\title{
An extragradient iterative scheme for common fixed point problems and variational inequality problems with applications
}

\author{
Adrian Petruşel, D.R. Sahu and Vidya Sagar
}

\begin{abstract}
In this paper, by combining a modified extragradient scheme with the viscosity approximation technique, an iterative scheme is developed for computing the common element of the set of fixed points of a sequence of asymptotically nonexpansive mappings and the set of solutions of the variational inequality problem for an $\alpha$-inverse strongly monotone mapping. We prove a strong convergence theorem for the sequences generated by this scheme and give some applications of our convergence theorem.
\end{abstract}

\section{Introduction}

Let $C$ be a nonempty subset of a real Hilbert space $H$ with inner product $\langle.,$. and norm $\|$.$\| , respectively. A mapping A: C \rightarrow H$ is called (see ([15]))

(i) monotone if

$$
\langle A u-A v, u-v\rangle \geq 0, \text { for all } u, v \in C
$$

(ii) $\eta$-strongly monotone if there exists a positive real number $\eta$ such that

$$
\langle A u-A v, u-v\rangle \geq \eta\|u-v\|^{2}, \quad \text { for all } u, v \in C ;
$$

Key Words: Modified extragradient method, viscosity approximation method, $\alpha$-inverse strongly monotone mapping, sequence of asymptotically nonexpansive mappings, variational inequalities, $\mathrm{AF}$ point property.

2010 Mathematics Subject Classification: Primary 47H10; Secondary 54H25.

Received: 5 May, 2014.

Revised: 20 June, 2014

Accepted: 27 June, 2014. 
AN EXTRAGRADIENT ITERATIVE SCHEME FOR COMMON FIXED POINT PROBLEMS AND VARIATIONAL INEQUALITY PROBLEMS WITH

(iii) $\alpha$-inverse strongly monotone if there exists a positive real number $\alpha$ such that

$$
\langle A u-A v, u-v\rangle \geq \alpha\|A u-A v\|^{2}, \text { for all } u, v \in C ;
$$

(iv) $k$-Lipschitzian if there exists $k>0$ such that

$$
\|A u-A v\| \leq k\|u-v\|, \quad \text { for all } u, v \in C ;
$$

(v) $k$-contraction if it is $k$-Lipschitzian with $k<1$;

(vi) nonexpansive if

$$
\|A u-A v\| \leq\|u-v\|, \quad \text { for all } u, v \in C .
$$

Let $C$ be a nonempty subset of a real Hilbert space $H$ and $\left\{S_{n}\right\}$ a sequence of mappings from $C$ into itself. Then the sequence $\left\{S_{n}\right\}_{n \in \mathbb{N}}$ is called a sequence of asymptotically nonexpansive mappings ([12]) on $C$ if there exists a sequence $\left\{k_{n}\right\}$ in $[1, \infty)$ with $\lim _{n \rightarrow \infty} k_{n}=1$ such that

$$
\left\|S_{n} u-S_{n} v\right\| \leq k_{n}\|u-v\| \text {, for all } u, v \in C \text { and } n \in \mathbb{N} \text {. }
$$

Let $C$ be a nonempty, closed, and convex subset of a real Hilbert space $H$. A variational inequality problem is the problem of finding $u \in C$ such that

$$
\langle A u, v-u\rangle \geq 0, \quad \text { for all } v \in C,
$$

where $A$ is a nonlinear mapping from $C$ into $H$. The set of solutions of the variational inequality problem (1.1) is denoted by $\Omega$. We denote by $F(S)$ the set of fixed points of mapping $S: C \rightarrow C$.

We give some examples of $\alpha$-inverse strongly monotone mappings. Let $H$ be a Hilbert space and $C$ a nonempty closed convex subset of $H$. If $T$ is a nonexpansive mapping from $C$ into itself, then $A:=I-T$ is $\frac{1}{2}$-inverse strongly monotone and $\Omega=F(T)$. Also, if $A$ is $\eta$-strongly monotone and $k$-Lipschitz, then $A$ is $\frac{\eta}{k^{2}}$-inverse strongly monotone. For the reverse implication, let us observe that there are examples of mappings which are inverse strongly monotone, but not strongly monotone. The metric projection $P_{C}$ is one of these, see also [16]. Recall that mapping $T: C \rightarrow C$ is called $\lambda$-strictly pseudocontractive on $C$ if there exists $\lambda \in[0,1)$ such that

$$
\|T x-T y\|^{2} \leq\|x-y\|^{2}+\lambda\|(I-T) x-(I-T) y\|^{2}, \text { for all } x, y \in C .
$$

Notice that if $T: C \rightarrow C$ is $\lambda$-strictly pseudocontractive, then the mapping $A:=I-T$ is $\frac{1-\lambda}{2}$-inverse strongly monotone. 
AN EXTRAGRADIENT ITERATIVE SCHEME FOR COMMON FIXED POINT PROBLEMS AND VARIATIONAL INEQUALITY PROBLEMS WITH

The variational inequality (1.1) was introduced by Stampacchia [14] in 1964 . It has been shown that a large class of problems arising in engineering and applied sciences ([8] and the references therein) can be studied in the framework of the variational inequalities. It is known that the element $u \in C$ is a solution of the variational inequality problem (1.1) if and only if $u$ satisfies the relation:

$$
u=P_{C}(u-\lambda A u),
$$

where $\lambda>0$ is a constant and $P_{C}$ is the metric projection mapping of $H$ onto $C$.

It is obvious that fixed point problems and variational inequality problems are equivalent. This approach shows that a variational inequality can be regarded as a fixed point problem and, in this respect, the following iterative method could be important, in order to solve approximatively a variational inequality problem:

For a given $u_{0} \in C$, compute $u_{n+1}$ by the iterative scheme:

$$
u_{n+1}=P_{C}\left(u_{n}-\lambda A u_{n}\right), \text { for } n=0,1,2, \ldots
$$

These ideas were the starting point of a large number of papers dealing with the problem of approximating the solution of a variational inequality problem, sometimes in connection to other related problems, such as the problem of finding and approximating the fixed points of a nonexpansive mapping.

Korpelevich [6] introduced an extragradient method and proved that the sequences generated by the extragradient method converge to the same point $z \in \Omega$.

Recently, Nadezhkina and Takahashi [7], Zeng and Yao [18] introduced new iterative schemes for finding an element of $F(S) \cap \Omega$ and obtained the weak and strong convergence theorems respectively. Chen, Zhang and Fan [2] introduced an iterative scheme by viscosity approximation method for finding a common element of the fixed point set of a nonexpansive operator and the solution set of a variational inequality problem and proved a strong convergence theorem.

More recently, Petruşel and Yao [10] introduced a modified extragradient scheme by viscosity approximation method and obtained a strong convergence result for an explicit scheme in a Hilbert space.

In this paper, inspired by Petruşel and Yao [10], we prove a strong convergence 
AN EXTRAGRADIENT ITERATIVE SCHEME FOR COMMON FIXED POINT PROBLEMS AND VARIATIONAL INEQUALITY PROBLEMS WITH

theorem for computation of the common element of the set of fixed points of a sequence of asymptotically nonexpansive mappings and the set of solutions of the variational inequality problem for an $\alpha$-inverse strongly monotone mapping. Our results generalize the result of Petruşel and Yao [10] to the case of a sequence of asymptotically nonexpansive mappings and extend the results of Nadezhkina and Takahashi [7], Zeng and Yao [18] and Chen, Zhang and Fan [2]. Other related results are given in [17]-[4].

\section{Preliminaries}

Throughout this paper, $H$ is a real Hilbert space with inner product $\langle\cdot, \cdot\rangle$ and norm $\|$.$\| . We denote by I$ the identity operator of $H$. Also, we denote by $\rightarrow$ and $\rightarrow$ the strong convergence and weak convergence, respectively. The symbol $\mathbb{N}$ stands for the set of all natural numbers. Let $C$ be a nonempty subset of $H$ and $\mathcal{S}:=\left\{S_{n}\right\}_{n \in \mathbb{N}}$ a sequence of self-mappings from $C$ into itself. We denote by $F(\mathcal{S})$ the set of common fixed points of the sequence $\mathcal{S}$, i.e., $F(\mathcal{S})=\bigcap_{n=1}^{\infty} F\left(S_{n}\right)$

Let $C$ be a nonempty closed convex subset of $H$. Then, for any $x \in H$, there exists a unique nearest point in $C$, denoted by $P_{C}(x)$, such that

$$
\left\|x-P_{C}(x)\right\| \leq\|x-y\|, \quad \text { for all } y \in C .
$$

The mapping $P_{C}$ is called the metric projection of $H$ onto $C$. We know that $P_{C}$ is a nonexpansive mapping of $H$ onto $C$. It is also known that $P_{C}$ is characterized by the following properties (see $[5,1])$ :

(A) $P_{C}(x) \in C$, for all $x \in H$;

(B) $\left\langle x-P_{C}(x), P_{C}(x)-y\right\rangle \geq 0$, for all $x \in H, y \in C$;

(C) $\|x-y\|^{2} \geq\left\|x-P_{C}(x)\right\|^{2}+\left\|y-P_{C}(x)\right\|^{2}$, for all $x \in H, y \in C$.

It is also known that $H$ satisfies the Opial property (see [1,9]), i.e., for any sequence $\left\{x_{n}\right\} \subset H$ with $x_{n} \rightarrow x$, the inequality

$$
\liminf _{n \rightarrow \infty}\left\|x_{n}-x\right\|<\liminf _{n \rightarrow \infty}\left\|x_{n}-y\right\|
$$

holds for every $y \in H$ with $y \neq x$.

A set-valued mapping $T: H \rightarrow 2^{H}$ is called monotone if, for all $x, y \in H$, $f \in T x$ and $g \in T y$, we have $\langle x-y, f-g\rangle \geq 0$. A monotone mapping $T: H \rightarrow 2^{H}$ is maximal if its graph $G(T)$ is not properly contained in the graph of any other monotone mapping. It is known that a monotone mapping $T$ is maximal if and only if the following implication holds: if for 
AN EXTRAGRADIENT ITERATIVE SCHEME FOR COMMON FIXED POINT PROBLEMS AND VARIATIONAL INEQUALITY PROBLEMS WITH

$(x, f) \in H \times H,\langle x-y, f-g\rangle \geq 0$ for all $(y, g) \in G(T)$, then $f \in T x$.

Let $A: C \rightarrow H$ be a monotone and $k$-Lipschitz continuous mapping and let $N_{C}(v)$ be the normal cone to $C$ at $v \in C$, i.e.,

$$
N_{C}(v)=\{w \in H:\langle v-y, w\rangle \geq 0 \text {, for all } y \in C\} .
$$

Define

$$
T v= \begin{cases}A v+N_{C}(v), & \text { if } v \in C, \\ \emptyset, & \text { if } v \notin C .\end{cases}
$$

Then $T$ is maximal monotone and $0 \in T v$ if and only if $v \in \Omega$, see ([11]).

We present now an important property of the $\alpha$-inverse strongly monotone mappings.

Lemma 1. ([16]) Let $C$ be a nonempty subset of a real Hilbert space $H$. Let $\alpha>0$ and $A: C \rightarrow H$ an $\alpha$-inverse strongly monotone. Then, $A$ is $\frac{1}{\alpha}$-Lipschitz continuous. Moreover, for all $u, v \in C$ and each $\lambda>0$, we have

$$
\begin{aligned}
\|(I-\lambda A) u-(I-\lambda A) v\|^{2}= & \|(u-v)-\lambda(A u-A v)\|^{2} \\
= & \|u-v\|^{2}-2 \lambda\langle u-v, A u-A v\rangle \\
& +\lambda^{2}\|A u-A v\|^{2} .
\end{aligned}
$$

As consequence, $(I-\lambda A)$ is a nonexpansive mapping from $C$ into $H$ if $\lambda \leq 2 \alpha$.

Now we state an existence result for the solution of the variational inequality problem for inverse strongly monotone mappings.

Theorem 1. ([16]) Let $C$ be a closed convex bounded subset of a real Hilbert space $H$ and let $A: C \rightarrow H$ be $\alpha$-inverse strongly monotone. Then $\Omega$ is non-empty.

In the proof of the main results, we need the following lemmas.

Lemma 2. (Schu [13]) Let $H$ be a real Hilbert space, let $\left\{\alpha_{n}\right\}$ be a sequence of real numbers such that $0<a \leq \alpha_{n} \leq b<1$, for all $n \in \mathbb{N}$ and let $\left\{v_{n}\right\}$ and $\left\{w_{n}\right\}$ be sequences in $H$ such that

$$
\limsup _{n \rightarrow \infty}\left\|v_{n}\right\| \leq c, \limsup _{n \rightarrow \infty}\left\|w_{n}\right\| \leq c \text { and } \lim _{n \rightarrow \infty}\left\|\alpha_{n} v_{n}+\left(1-\alpha_{n}\right) w_{n}\right\|=c
$$


AN EXTRAGRADIENT ITERATIVE SCHEME FOR COMMON FIXED POINT PROBLEMS AND VARIATIONAL INEQUALITY PROBLEMS WITH

for some $c \geq 0$. Then, $\lim _{n \rightarrow \infty}\left\|v_{n}-w_{n}\right\|=0$.

Lemma 3. (Xu [17]) Let $\left\{\alpha_{n}\right\}_{n=0}^{\infty}$ be a sequence of non negative real numbers satisfying the inequality

$$
\alpha_{n+1} \leq\left(1-\gamma_{n}\right) \alpha_{n}+\gamma_{n} \beta_{n}, \quad \text { for all } n \in \mathbb{N},
$$

where $\left\{\gamma_{n}\right\}_{n=1}^{\infty}$ and $\left\{\beta_{n}\right\}_{n=1}^{\infty}$ are sequences of real numbers which satisfy the conditions:

(i) $\left\{\gamma_{n}\right\}_{n=1}^{\infty} \subset(0,1)$ and $\sum_{n=1}^{\infty} \gamma_{n}=\infty$;

(ii) $\limsup _{n \rightarrow \infty} \frac{\beta_{n}}{\gamma_{n}} \leq 0$ or $\sum_{n=1}^{\infty}\left|\beta_{n}\right|<\infty$.

Then $\lim _{n \rightarrow \infty} \alpha_{n}=0$.

Lemma 4. ([3]) Assume that $S$ is an asymptotically nonexpansive self-mapping of a nonempty closed convex subset $C$ of a real Hilbert space $H$. Then $I-S$ is demiclosed, i.e., if $\left\{x_{n}\right\}$ is a sequence in $C$ weakly converging to some $x \in C$ and the sequence $\left\{(I-S) x_{n}\right\}$ strongly converges to 0 , then $x \in F(S)$.

AF point property ([12]) Let $C$ be a nonempty subset of a real Hilbert space $H$, and let $\mathcal{S}:=\left\{S_{n}\right\}$ be a sequence of self-mappings on $C$. A sequence $\left\{x_{n}\right\}$ in $C$ is said to have the approximate fixed point property (in short AF point property) for $\left\{S_{n}\right\}$ if $\lim _{n \rightarrow \infty}\left\|x_{n}-S_{n} x_{n}\right\|=0$.

Condition $\mathcal{D}([12])$ Let $C$ be a nonempty closed convex subset of a real Hilbert space $H$ and $\mathcal{S}:=\left\{S_{n}\right\}$ a sequence of self-mappings on $C$. A family $\left\{I-S_{n}\right\}$ is said to be demi-closed at zero if for every bounded sequence $\left\{x_{n}\right\}$ in $C$, the following condition holds:

$$
\left\{x_{n}-S_{n} x_{n}\right\} \rightarrow 0 \Rightarrow w_{w}\left(x_{n}\right) \subset F(\mathcal{S}),
$$

where $w_{w}\left(x_{n}\right)$ is the set of weak cluster points of the sequence $\left\{x_{n}\right\}$.

\section{$3 \quad$ Main results}

Theorem 2. Let $C$ be a nonempty, closed and convex subset of a real Hilbert space $H$. Let $A: C \rightarrow H$ be an $\alpha$-inverse strongly monotone mapping and $\mathcal{S}:=$ $\left\{S_{n}\right\}$ a sequence of asymptotically nonexpansive mappings from $C$ into itself with sequence $\left\{k_{n}\right\}$ such that $F(\mathcal{S}) \cap \Omega \neq \emptyset$. Assume that $\mathcal{S}$ satisfies condition 
AN EXTRAGRADIENT ITERATIVE SCHEME FOR COMMON FIXED POINT PROBLEMS AND VARIATIONAL INEQUALITY PROBLEMS WITH

(D) and $f: C \rightarrow C$ is a $k$-contraction. For arbitrary $x_{1} \in C$, consider the sequences $\left\{x_{n}\right\}$ and $\left\{y_{n}\right\}$ generated by the following iterative process:

$$
\left\{\begin{array}{l}
x_{1} \in C, \\
y_{n}=P_{C}\left(x_{n}-\lambda_{n} A x_{n}\right), \\
x_{n+1}=\alpha_{n} f\left(x_{n}\right)+\left(1-\alpha_{n}\right) S_{n} P_{C}\left(x_{n}-\lambda_{n} A y_{n}\right), \text { for all } n \in \mathbb{N},
\end{array}\right.
$$

where $\left\{\alpha_{n}\right\}$ and $\left\{\lambda_{n}\right\}$ are two sequences of positive numbers with $\left\{\alpha_{n}\right\} \subset(0, \bar{\alpha})$ and $\left\{\lambda_{n}\right\} \subset[a, b]$, with $0<a<b<\alpha(1-\delta)$ (forsome $\bar{\alpha}, \delta \in(0,1)$ ) satisfying the conditions:

(i) $\lim _{n \rightarrow \infty} \alpha_{n}=0, \sum_{n=1}^{\infty} \alpha_{n}=\infty$;

(ii) $\sum_{n=1}^{\infty}\left|\alpha_{n+1}-\alpha_{n}\right|<\infty$;

(iii) $\sum_{n=1}^{\infty}\left|\lambda_{n+1}-\lambda_{n}\right|<\infty$;

(iv) $\lim _{n \rightarrow \infty} \frac{\left\|S_{n} t_{n}-S_{n+1} t_{n}\right\|}{\alpha_{n+1}}=0$;

(v) $\lim _{n \rightarrow \infty} \frac{k_{n}-1}{\alpha_{n}}=0$.

Then, the sequences $\left\{x_{n}\right\}$ and $\left\{y_{n}\right\}$ converge strongly to the same point $p$, such that $p$ is the unique solution in $F(\mathcal{S}) \cap \Omega$ of the following variational inequality:

$$
\langle f(p)-p, y-p\rangle \leq 0 \text { for all } y \in F(\mathcal{S}) \cap \Omega .
$$

Proof. Denote $t_{n}:=P_{C}\left(x_{n}-\lambda_{n} A y_{n}\right), \forall n \in \mathbb{N}$ and let $u \in F(\mathcal{S}) \cap \Omega$. Then $u=P_{C}\left(u-\lambda_{n} A u\right)$. We proceed in the following steps.

Step 1. $\left\{x_{n}\right\}$ is bounded.

Taking $x:=x_{n}-\lambda_{n} A y_{n}$ and $y:=u$ in relation (C), we have

$$
\begin{aligned}
\left\|t_{n}-u\right\|^{2} \leq & \left\|x_{n}-\lambda_{n} A y_{n}-u\right\|^{2}-\left\|x_{n}-\lambda_{n} A y_{n}-t_{n}\right\|^{2} \\
= & \left\|x_{n}-u\right\|^{2}-2 \lambda_{n}\left\langle A y_{n}, x_{n}-u\right\rangle+\lambda_{n}^{2}\left\|A y_{n}\right\|^{2}-\left\|x_{n}-t_{n}\right\|^{2} \\
& +2 \lambda_{n}\left\langle A y_{n}, x_{n}-t_{n}\right\rangle-\lambda_{n}^{2}\left\|A y_{n}\right\|^{2} \\
= & \left\|x_{n}-u\right\|^{2}+2 \lambda_{n}\left\langle A y_{n}, u-t_{n}\right\rangle-\left\|x_{n}-t_{n}\right\|^{2} \\
= & \left\|x_{n}-u\right\|^{2}-\left\|x_{n}-t_{n}\right\|^{2}-2 \lambda_{n}\left\langle A y_{n}-A u, y_{n}-u\right\rangle \\
& -2 \lambda_{n}\left\langle A u, y_{n}-u\right\rangle+2 \lambda_{n}\left\langle A y_{n}, y_{n}-t_{n}\right\rangle \\
\leq & \left\|x_{n}-u\right\|^{2}-\left\|x_{n}-y_{n}\right\|^{2}-\left\|y_{n}-t_{n}\right\|^{2} \\
& +2\left\langle x_{n}-\lambda_{n} A y_{n}-y_{n}, t_{n}-y_{n}\right\rangle .
\end{aligned}
$$


AN EXTRAGRADIENT ITERATIVE SCHEME FOR COMMON FIXED POINT PROBLEMS AND VARIATIONAL INEQUALITY PROBLEMS WITH

From (B), we obtain

$$
\begin{aligned}
\left\langle x_{n}-\lambda_{n} A y_{n}-y_{n}, t_{n}-y_{n}\right\rangle= & \left\langle x_{n}-\lambda_{n} A x_{n}-y_{n}, t_{n}-y_{n}\right\rangle \\
& +\left\langle\lambda_{n} A x_{n}-\lambda_{n} A y_{n}, t_{n}-y_{n}\right\rangle \\
= & -\left\langle x_{n}-\lambda_{n} A x_{n}-y_{n}, y_{n}-t_{n}\right\rangle \\
& +\left\langle\lambda_{n} A x_{n}-\lambda_{n} A y_{n}, t_{n}-y_{n}\right\rangle \\
\leq & \left\langle\lambda_{n} A x_{n}-\lambda_{n} A y_{n}, t_{n}-y_{n}\right\rangle \\
\leq & \frac{\lambda_{n}}{\alpha}\left\|x_{n}-y_{n}\right\|\left\|t_{n}-y_{n}\right\| .
\end{aligned}
$$

Hence

$$
\begin{aligned}
\left\|t_{n}-u\right\|^{2} \leq & \left\|x_{n}-u\right\|^{2}-\left\|x_{n}-y_{n}\right\|^{2}-\left\|y_{n}-t_{n}\right\|^{2} \\
& +2\left\langle x_{n}-\lambda_{n} A y_{n}-y_{n}, t_{n}-y_{n}\right\rangle \\
\leq & \left\|x_{n}-u\right\|^{2}-\left\|x_{n}-y_{n}\right\|^{2}-\left\|y_{n}-t_{n}\right\|^{2} \\
& +\frac{\lambda_{n}{ }^{2}}{\alpha^{2}}\left\|x_{n}-y_{n}\right\|^{2}+\left\|t_{n}-y_{n}\right\|^{2} \\
= & \left\|x_{n}-u\right\|^{2}+\left(\frac{\lambda_{n}{ }^{2}}{\alpha^{2}}-1\right)\left\|x_{n}-y_{n}\right\|^{2} \\
\leq & \left\|x_{n}-u\right\|^{2} .
\end{aligned}
$$

From (3.1), we have

$$
\begin{aligned}
\left\|x_{n+1}-u\right\| \leq & \left\|\alpha_{n} f\left(x_{n}\right)+\left(1-\alpha_{n}\right) S_{n} t_{n}-u\right\| \\
\leq & \alpha_{n}\left\|f\left(x_{n}\right)-u\right\|+\left(1-\alpha_{n}\right)\left\|S_{n} t_{n}-u\right\| \\
\leq & \alpha_{n}\left\|f\left(x_{n}\right)-f(u)\right\|+\alpha_{n}\|f(u)-u\|+\left(1-\alpha_{n}\right)\left\|S_{n} t_{n}-S u\right\| \\
\leq & \alpha_{n} k\left\|x_{n}-u\right\|+\alpha_{n}\|f(u)-u\|+k_{n}\left(1-\alpha_{n}\right)\left\|t_{n}-u\right\| \\
\leq & \alpha_{n} k\left\|x_{n}-u\right\|+\alpha_{n}\|f(u)-u\|+k_{n}\left(1-\alpha_{n}\right)\left\|x_{n}-u\right\| \\
= & {\left[1-\alpha_{n}(1-k)\right]\left\|x_{n}-u\right\|+\alpha_{n}\|f(u)-u\| } \\
& +\left(1-\alpha_{n}\right)\left(k_{n}-1\right)\left\|x_{n}-u\right\| \\
= & {\left[1-\alpha_{n}(1-k)\right]\left\|x_{n}-u\right\|+\left(1-\alpha_{n}\right)\left(k_{n}-1\right)\left\|x_{n}-u\right\|+\mu_{n}, }
\end{aligned}
$$

where $\mu_{n}=\alpha_{n}\|f(u)-u\|$. Note $\lim _{n \rightarrow \infty} \frac{k_{n}-1}{\alpha_{n}}=0$ and $\lim _{n \rightarrow \infty} \alpha_{n}=0$, so there exist two constants $\beta \in(0,1)$ with $(\beta-k) \in(0,1)$ and $K_{1}>0$ such that $\frac{k_{n}-1}{\alpha_{n}} \leq \frac{1-\beta}{1-\alpha_{n}}$ and $\frac{\mu_{n}}{\alpha_{n}} \leq K_{1}$ for all $n \in \mathbb{N}$. Hence

$$
\begin{aligned}
\left\|x_{n+1}-u\right\| & \leq\left[1-(\beta-k) \alpha_{n}\right]\left\|x_{n}-u\right\|+\alpha_{n} K_{1} \\
& \leq \max \left\{\left\|x_{n}-u\right\|, \frac{K_{1}}{\beta-k}\right\} .
\end{aligned}
$$


AN EXTRAGRADIENT ITERATIVE SCHEME FOR COMMON FIXED POINT PROBLEMS AND VARIATIONAL INEQUALITY PROBLEMS WITH

It follows that $\left\{x_{n}\right\}$ is bounded.

Step 2. $\left\|x_{n+1}-x_{n}\right\| \rightarrow 0$ as $n \rightarrow \infty$.

By Step 1, $\left\{x_{n}\right\}$ is bounded. So $\left\{f\left(x_{n}\right)\right\},\left\{A x_{n}\right\},\left\{t_{n}\right\},\left\{A t_{n}\right\},\left\{S_{n} t_{n}\right\}$ are bounded. Observe that

$$
\begin{aligned}
\left\|x_{n+1}-x_{n}\right\|= & \|\left(\alpha_{n}-\alpha_{n-1}\right)\left[f\left(x_{n-1}\right)-S_{n-1} t_{n-1}\right] \\
& +\left(1-\alpha_{n}\right)\left(S_{n} t_{n}-S_{n-1} t_{n-1}\right)+\alpha_{n}\left[f\left(x_{n}\right)-f\left(x_{n-1}\right)\right] \| \\
\leq & \left|\alpha_{n}-\alpha_{n-1}\right|\left\|f\left(x_{n-1}\right)-S_{n-1} t_{n-1}\right\| \\
& +\left(1-\alpha_{n}\right)\left\|S_{n} t_{n}-S_{n-1} t_{n-1}\right\|+\alpha_{n}\left\|f\left(x_{n}\right)-f\left(x_{n-1}\right)\right\| \\
\leq & \left|\alpha_{n}-\alpha_{n-1}\right|\left\|f\left(x_{n-1}\right)-S_{n-1} t_{n-1}\right\| \\
& +\left(1-\alpha_{n}\right)\left\|S_{n} t_{n}-S_{n-1} t_{n-1}\right\|+\alpha_{n} k\left\|x_{n}-x_{n-1}\right\| \\
\leq & \left|\alpha_{n}-\alpha_{n-1}\right|\left\|f\left(x_{n-1}\right)-S_{n-1} t_{n-1}\right\| \\
& +k_{n}\left(1-\alpha_{n}\right)\left\|t_{n}-t_{n-1}\right\|+\alpha_{n} k\left\|x_{n}-x_{n-1}\right\|+\left(1-\alpha_{n}\right) \epsilon_{n-1},
\end{aligned}
$$

where $\epsilon_{n-1}=\left\|S_{n} t_{n-1}-S_{n-1} t_{n-1}\right\|$. Since $\lambda_{n}<\alpha(1-\delta)<2 \alpha$, from Proposition 1 , we have

$$
\begin{aligned}
\left\|t_{n+1}-t_{n}\right\| & =\left\|P_{C}\left(x_{n+1}-\lambda_{n+1} A y_{n+1}\right)-P_{C}\left(x_{n}-\lambda_{n} A y_{n}\right)\right\| \\
& \leq\left\|x_{n+1}-\lambda_{n+1} A y_{n+1}-x_{n}+\lambda_{n} A y_{n}\right\| \\
& \leq\left\|x_{n+1}-x_{n}\right\|+\left|\lambda_{n+1}-\lambda_{n}\right|\left\|A y_{n}\right\| .
\end{aligned}
$$

Hence

$$
\begin{aligned}
\left\|x_{n+1}-x_{n}\right\| \leq & {\left[k_{n}\left(1-\alpha_{n}\right)+k \alpha_{n}\right]\left\|x_{n}-x_{n-1}\right\| } \\
& +\left|\alpha_{n}-\alpha_{n-1}\right|\left\|f\left(x_{n-1}\right)-S_{n-1} t_{n-1}\right\| \\
& +k_{n}\left(1-\alpha_{n}\right)\left|\lambda_{n}-\lambda_{n-1}\right|\left\|A y_{n-1}\right\|+\left(1-\alpha_{n}\right) \epsilon_{n-1} \\
\leq & {\left[k_{n}\left(1-\alpha_{n}\right)+k \alpha_{n}\right]\left\|x_{n}-x_{n-1}\right\| } \\
& +\left|\alpha_{n}-\alpha_{n-1}\right| L+\left|\lambda_{n}-\lambda_{n-1}\right| M+\left(1-\alpha_{n}\right) \epsilon_{n-1} \\
\leq & {\left[1-(1-k) \alpha_{n}\right]\left\|x_{n}-x_{n-1}\right\|+\left(k_{n}-1\right) N } \\
& +\left|\alpha_{n}-\alpha_{n-1}\right| L+\left|\lambda_{n}-\lambda_{n-1}\right| M+\epsilon_{n-1},
\end{aligned}
$$

where $L=\sup _{n \in \mathbb{N}}\left\|f\left(x_{n}\right)-S_{n} t_{n}\right\|, M=\sup _{n \in \mathbb{N}}\left\|A y_{n}\right\|$ and $N=\sup _{n \in \mathbb{N}}\left\|x_{n}-x_{n+1}\right\|$. Note $\lim _{n \rightarrow \infty} \frac{k_{n}-1}{\alpha_{n}}=0$ and $\lim _{n \rightarrow \infty} \frac{\epsilon_{n}}{\alpha_{n+1}}=0$. By Lemma 3 , we obtain $\left\|x_{n+1}-x_{n}\right\| \rightarrow 0$ as $n \rightarrow \infty$. 
AN EXTRAGRADIENT ITERATIVE SCHEME FOR COMMON FIXED POINT PROBLEMS AND VARIATIONAL INEQUALITY PROBLEMS WITH

Step 3. $\left\|x_{n}-y_{n}\right\| \rightarrow 0$ and $\left\|t_{n}-y_{n}\right\| \rightarrow 0$ as $n \rightarrow \infty$.

From (3.1), we have

$$
\begin{aligned}
\left\|x_{n+1}-u\right\|^{2}= & \left\|\alpha_{n} f\left(x_{n}\right)+\left(1-\alpha_{n}\right) S_{n} t_{n}-u\right\|^{2} \\
= & \left\|\alpha_{n}\left(f\left(x_{n}\right)-f(u)\right)+\alpha_{n}(f(u)-u)+\left(1-\alpha_{n}\right)\left(S_{n} t_{n}-u\right)\right\|^{2} \\
\leq & \alpha_{n}\left[\left\|f\left(x_{n}\right)-f(u)\right\|+\|f(u)-u\|\right]^{2} \\
& +\left(1-\alpha_{n}\right)\left\|S_{n} t_{n}-u\right\|^{2} \\
\leq & \alpha_{n}\left[k\left\|x_{n}-u\right\|+\|f(u)-u\|\right]^{2}+k_{n}^{2}\left(1-\alpha_{n}\right)\left\|t_{n}-u\right\|^{2} \\
\leq & k^{2} \alpha_{n}\left\|x_{n}-u\right\|^{2}+\alpha_{n}\left[2 k\left\|x_{n}-u\right\|\|f(u)-u\|\right. \\
& \left.+\|f(u)-u\|^{2}\right]+k_{n}^{2}\left(1-\alpha_{n}\right)\left[\left\|x_{n}-u\right\|^{2}\right. \\
& \left.+\left(\frac{\lambda_{n}^{2}}{\alpha^{2}}-1\right)\left\|x_{n}-y_{n}\right\|^{2}\right] \\
\leq & {\left[k^{2} \alpha_{n}+k_{n}^{2}\left(1-\alpha_{n}\right)\right]\left\|x_{n}-u\right\|^{2} } \\
& +\alpha_{n}\left[2 k\left\|x_{n}-u\right\|\|f(u)-u\|+\|f(u)-u\|^{2}\right] \\
& +k_{n}^{2}\left(1-\alpha_{n}\right)\left(\frac{\lambda_{n}^{2}}{\alpha^{2}}-1\right)\left\|x_{n}-y_{n}\right\|^{2} .
\end{aligned}
$$

Hence

$$
\begin{aligned}
&(1-\bar{\alpha})\left(2 \delta-\delta^{2}\right)\left\|x_{n}-y_{n}\right\|^{2} \leq k_{n}^{2}\left(1-\alpha_{n}\right)\left(1-\frac{\lambda_{n}^{2}}{\alpha^{2}}\right)\left\|x_{n}-y_{n}\right\|^{2} \\
& \leq \quad {\left[k^{2} \alpha_{n}+k_{n}^{2}\left(1-\alpha_{n}\right)\right]\left\|x_{n}-u\right\|^{2}-\left\|x_{n+1}-u\right\|^{2} } \\
&+\alpha_{n}\left[2 k\left\|x_{n}-u\right\|\|f(u)-u\|+\|f(u)-u\|^{2}\right] \\
& \leq {\left[k^{2} \alpha_{n}+\left(1-\alpha_{n}\right)\right]\left\|x_{n}-u\right\|^{2}-\left\|x_{n+1}-u\right\|^{2} } \\
&+\left(1-\alpha_{n}\right)\left(k_{n}^{2}-1\right)\left\|x_{n}-u\right\|^{2} \\
&+\alpha_{n}\left[2 k\left\|x_{n}-u\right\|\|f(u)-u\|+\|f(u)-u\|^{2}\right] \\
& \leq\left(\left\|x_{n}-u\right\|^{2}-\left\|x_{n+1}-u\right\|^{2}\right) \\
&+\left(k_{n}-1\right)\left(k_{n}+1\right)\left\|x_{n}-u\right\|^{2} \\
&+\alpha_{n}\left[2 k\left\|x_{n}-u\right\|\|f(u)-u\|+\|f(u)-u\|^{2}\right] \\
&= {\left[\left(\left\|x_{n}-u\right\|-\left\|x_{n+1}-u\right\|\right)\left(\left\|x_{n}-u\right\|+\left\|x_{n+1}-u\right\|\right)\right] } \\
&+\left(k_{n}-1\right) \sup _{i \in \mathbb{N}}\left(k_{i}+1\right) R^{2} \\
&+\alpha_{n}\left[2 k\left\|x_{n}-u\right\|\|f(u)-u\|+\|f(u)-u\|^{2}\right] \\
& \leq 2\left\|x_{n}-x_{n+1}\right\| R+\left(k_{n}-1\right) \sup _{i \in \mathbb{N}}\left(k_{i}+1\right) R^{2} \\
&+\alpha_{n}\left[2 R k\|f(u)-u\|+\|f(u)-u\|^{2}\right],
\end{aligned}
$$


AN EXTRAGRADIENT ITERATIVE SCHEME FOR COMMON FIXED POINT

where $R$ is a positive constant such that $\left\|x_{n}-u\right\| \leq R$ for all $n \in \mathbb{N}$. Note $k_{n} \rightarrow 1,\left\|x_{n+1}-x_{n}\right\| \rightarrow 0$ and $\alpha_{n} \rightarrow 0$ as $n \rightarrow \infty$, we have, $\left\|x_{n}-y_{n}\right\| \rightarrow 0$ as $n \rightarrow \infty$.

Observe that

$$
\begin{aligned}
\left\|y_{n}-t_{n}\right\| & =\left\|P_{C}\left(x_{n}-\lambda_{n} A x_{n}\right)-P_{C}\left(x_{n}-\lambda_{n} A y_{n}\right)\right\| \\
& \leq\left\|\left(x_{n}-\lambda_{n} A x_{n}\right)-\left(x_{n}-\lambda_{n} A y_{n}\right)\right\| \\
& \leq \frac{\lambda_{n}}{\alpha}\left\|x_{n}-y_{n}\right\| \rightarrow 0 \text { as } n \rightarrow \infty .
\end{aligned}
$$

Step 4. $\limsup _{n \rightarrow \infty}\left\langle f(p)-p, S_{n} t_{n}-p\right\rangle \leq 0$, where $p=P_{F(\delta) \cap \Omega} f(p)$.

For $u \in F(\mathcal{S}) \cap \Omega$, we have

$$
\begin{aligned}
\| S_{n} y_{n} & -x_{n+1}\|\leq\| S_{n} y_{n}-S_{n} t_{n}\|+\| S_{n} t_{n}-x_{n+1} \| \\
& \leq k_{n}\left\|y_{n}-t_{n}\right\|+\alpha_{n}\left\|S_{n} t_{n}-f\left(x_{n}\right)\right\| \\
& \leq k_{n}\left\|y_{n}-t_{n}\right\|+\alpha_{n}\left[\left\|S_{n} t_{n}-u\right\|+\left\|u-f\left(x_{n}\right)\right\|\right] \\
& \leq k_{n}\left\|y_{n}-t_{n}\right\|+\alpha_{n}\left[k_{n}\left\|t_{n}-u\right\|+\left\|u-f\left(x_{n}\right)\right\|\right] \\
& \leq k_{n}\left\|y_{n}-t_{n}\right\|+\alpha_{n}\left[k_{n} \max \left\{\left\|x_{n}-u\right\|, \frac{K_{1}}{\beta-k}\right\}+\left\|u-f\left(x_{n}\right)\right\|\right] .
\end{aligned}
$$

Hence $\left\|S_{n} y_{n}-x_{n+1}\right\| \rightarrow 0$ as $n \rightarrow \infty$. Note that $\left\|x_{n}-y_{n}\right\| \rightarrow 0$ as $n \rightarrow \infty$, we have

$$
\begin{aligned}
\left\|S_{n} x_{n}-x_{n}\right\| \leq\left\|S_{n} x_{n}-S_{n} y_{n}\right\|+\left\|S_{n} y_{n}-x_{n+1}\right\|+\left\|x_{n}-x_{n+1}\right\| \\
\leq \quad k_{n}\left\|x_{n}-y_{n}\right\|+\left\|S_{n} y_{n}-x_{n+1}\right\|+\left\|x_{n}-x_{n+1}\right\| \rightarrow 0 \text { as } n \rightarrow \infty
\end{aligned}
$$

and

$$
\begin{aligned}
\left\|S_{n} t_{n}-t_{n}\right\| & \leq\left\|S_{n} t_{n}-S_{n} x_{n}\right\|+\left\|S_{n} x_{n}-x_{n}\right\|+\left\|x_{n}-t_{n}\right\| \\
& \leq k_{n}\left\|t_{n}-x_{n}\right\|+\left\|S_{n} x_{n}-x_{n}\right\|+\left\|x_{n}-t_{n}\right\| \\
& \leq\left\|S_{n} x_{n}-x_{n}\right\|+\left(1+k_{n}\right)\left\|x_{n}-t_{n}\right\| \rightarrow 0 \text { as } n \rightarrow \infty .
\end{aligned}
$$

Now, let us choose a subsequence $\left\{t_{n_{i}}\right\}$ of $\left\{t_{n}\right\}$ such that

$$
\limsup _{n \rightarrow \infty}\left\langle f(p)-p, S_{n} t_{n}-p\right\rangle=\lim _{i \rightarrow \infty}\left\langle f(p)-p, S_{n_{i}} t_{n_{i}}-p\right\rangle .
$$

For the convenience we will denote this subsequence by $\left\{t_{n}\right\}$ too. As $\left\{t_{n}\right\}$ is bounded, we have that a subsequence $\left\{t_{n_{i}}\right\}$ of $\left\{t_{n}\right\}$ converges weakly to some $z \in C$. Since $\left\|x_{n}-y_{n}\right\| \rightarrow 0$ and $\left\|y_{n}-t_{n}\right\| \rightarrow 0$ as $n \rightarrow \infty$, we have that 
AN EXTRAGRADIENT ITERATIVE SCHEME FOR COMMON FIXED POINT PROBLEMS AND VARIATIONAL INEQUALITY PROBLEMS WITH

$\left\{x_{n_{i}}\right\}$ and $\left\{y_{n_{i}}\right\}$ converges weakly to $z \in C$. Also, since $\lim _{n \rightarrow \infty}\left\|S_{n} t_{n}-t_{n}\right\|=0$, by condition $(\mathcal{D})$, we get that $z \in F(\mathcal{S})$. From the above arguments, we have

$$
\limsup _{n \rightarrow \infty}\left\langle f(p)-p, S_{n} t_{n}-p\right\rangle=\lim _{i \rightarrow \infty}\left\langle f(p)-p, S_{n_{i}} t_{n_{i}}-p\right\rangle=\langle f(p)-p, z-p\rangle .
$$

Notice now that, in order to prove that $\limsup _{n \rightarrow \infty}\left\langle f(p)-p, S_{n} t_{n}-p\right\rangle \leq 0$, it suffices to show that $z \in F(\mathcal{S}) \cap \Omega$.

Now, let us show that $z \in \Omega$. Let

$$
T v= \begin{cases}A v+N_{C}(v), & \text { if } v \in C, \\ \emptyset, & \text { if } v \notin C .\end{cases}
$$

$T$ is maximal monotone and $0 \in T v$ if and only if $v \in \Omega$. Let $(v, w) \in G(T)$. Then, we have $w \in T v=A v+N_{C}(v)$ and hence $w-A v \in N_{C}(v)$. Thus, we have $\langle v-u, w-A v\rangle \geq 0$, for all $u \in C$.

On the other hand, from $t_{n}:=P_{C}\left(x_{n}-\lambda_{n} A y_{n}\right)$ and $v \in C$, we have $\left\langle x_{n}-\lambda_{n} A y_{n}-t_{n}, t_{n}-v\right\rangle \geq 0$, and hence $\left\langle v-t_{n}, \frac{t_{n}-x_{n}}{\lambda_{n}}+A y_{n}\right\rangle \geq 0$. Therefore, from $w-A v \in N_{C}(v)$ and $t_{n_{i}} \in C$, We have

$$
\begin{aligned}
\left\langle v-t_{n_{i}}, w\right\rangle \geq & \left\langle v-t_{n_{i}}, A v\right\rangle \\
\geq & \left\langle v-t_{n_{i}}, A v\right\rangle-\left\langle v-t_{n_{i}}, \frac{t_{n_{i}}-x_{n_{i}}}{\lambda_{n_{i}}}+A y_{n_{i}}\right\rangle \\
= & \left\langle v-t_{n_{i}}, A v-A t_{n_{i}}\right\rangle+\left\langle v-t_{n_{i}}, A t_{n_{i}}-A y_{n_{i}}\right\rangle \\
& -\left\langle v-t_{n_{i}}, \frac{t_{n_{i}}-x_{n_{i}}}{\lambda_{n_{i}}}\right\rangle \\
\geq & \left\langle v-t_{n_{i}}, A t_{n_{i}}-A y_{n_{i}}\right\rangle-\left\langle v-t_{n_{i}}, \frac{t_{n_{i}}-x_{n_{i}}}{\lambda_{n_{i}}}\right\rangle .
\end{aligned}
$$

Hence, letting $n_{i} \rightarrow \infty$ we obtain $\langle v-z, w\rangle \geq 0$. Thus, $z \in T^{-1} 0$ together with the maximal monotonicity of $T$ imply $z \in \Omega$.

Step 5. $x_{n} \rightarrow p$ as $n \rightarrow \infty$, where $p=P_{F(\mathcal{S}) \cap \Omega} f(p)$. i.e., $p$ is the unique solution in $F(\mathcal{S}) \cap \Omega$ of the variational inequality

$$
\langle f(p)-p, y-p\rangle \leq 0, \text { for all } y \in F(\mathcal{S}) \cap \Omega .
$$


AN EXTRAGRADIENT ITERATIVE SCHEME FOR COMMON FIXED POINT PROBLEMS AND VARIATIONAL INEQUALITY PROBLEMS WITH

We have

$$
\begin{aligned}
\left\|x_{n+1}-p\right\|^{2}= & \left\|\alpha_{n}\left(f\left(x_{n}\right)-p\right)+\left(1-\alpha_{n}\right)\left(S_{n} t_{n}-p\right)\right\|^{2} \\
= & \alpha_{n}^{2}\left\|f\left(x_{n}\right)-p\right\|^{2}+\left(1-\alpha_{n}\right)^{2}\left\|S_{n} t_{n}-p\right\|^{2} \\
& +2 \alpha_{n}\left(1-\alpha_{n}\right)\left\langle f\left(x_{n}\right)-p, S_{n} t_{n}-p\right\rangle \\
\leq & \alpha_{n}^{2}\left\|f\left(x_{n}\right)-p\right\|^{2}+k_{n}^{2}\left(1-\alpha_{n}\right)^{2}\left\|t_{n}-p\right\|^{2} \\
& +2 \alpha_{n}\left(1-\alpha_{n}\right)\left\langle f\left(x_{n}\right)-f(p), S_{n} t_{n}-p\right\rangle \\
& +2 \alpha_{n}\left(1-\alpha_{n}\right)\left\langle f(p)-p, S_{n} t_{n}-p\right\rangle \\
\leq & \alpha_{n}^{2}\left\|f\left(x_{n}\right)-p\right\|^{2}+k_{n}^{2}\left(1-\alpha_{n}\right)^{2}\left\|x_{n}-p\right\|^{2} \\
& +2 k k_{n} \alpha_{n}\left(1-\alpha_{n}\right)\left\|x_{n}-p\right\|\left\|t_{n}-p\right\| \\
& +2 \alpha_{n}\left(1-\alpha_{n}\right)\left\langle f(p)-p, S_{n} t_{n}-p\right\rangle \\
\leq & \alpha_{n}^{2}\left\|f\left(x_{n}\right)-p\right\|^{2}+k_{n}^{2}\left(1-\alpha_{n}\right)^{2}\left\|x_{n}-p\right\|^{2} \\
& +2 k k_{n} \alpha_{n}\left(1-\alpha_{n}\right)\left\|x_{n}-p\right\|^{2} \\
& +2 \alpha_{n}\left(1-\alpha_{n}\right)\left\langle f(p)-p, S_{n} t_{n}-p\right\rangle \\
\leq & \alpha_{n}^{2}\left\|f\left(x_{n}\right)-p\right\|^{2}+\left(1-\alpha_{n}\right)^{2}\left\|x_{n}-p\right\|^{2} \\
& +2 k \alpha_{n}\left(1-\alpha_{n}\right)\left\|x_{n}-p\right\|^{2} \\
& +2 \alpha_{n}\left(1-\alpha_{n}\right)\left\langle f(p)-p, S_{n} t_{n}-p\right\rangle+\left(k_{n-1}\right) \Gamma \\
= & {\left[1-\alpha_{n}\left\{2-\alpha_{n}-2 k\left(1-\alpha_{n}\right)\right\}\right]\left\|x_{n}-p\right\|^{2}+\alpha_{n}^{2}\left\|f\left(x_{n}\right)-p\right\|^{2} } \\
& +2 \alpha_{n}\left(1-\alpha_{n}\right)\left\langle f(p)-p, S_{n} t_{n}-p\right\rangle+\left(k_{n-1}\right) \Gamma \\
= & \left(1-\gamma_{n}\right)\left\|x_{n}-p\right\|^{2}+\gamma_{n} \beta_{n}+\left(k_{n-1}\right) \Gamma
\end{aligned}
$$

where $\Gamma>0$ is some constant, $\gamma_{n}=\alpha_{n}\left(2-\alpha_{n}-2 k\left(1-\alpha_{n}\right)\right)$ and

$$
\beta_{n}=\frac{\alpha_{n}\left\|f\left(x_{n}\right)-p\right\|^{2}+2\left(1-\alpha_{n}\right)\left\langle f(p)-p, S_{n} t_{n}-p\right\rangle}{2-\alpha_{n}-2 k\left(1-\alpha_{n}\right)} .
$$

Since $k_{n} \rightarrow 1$ and $\gamma_{n} \rightarrow 0$ as $n \rightarrow \infty, \sum_{n=1}^{\infty} \gamma_{n}=\infty$ and $\limsup _{n \rightarrow \infty} \beta_{n} \leq 0$, by applying Lemma 3 and using Step 4 , we obtain $x_{n} \rightarrow p$ as $n \rightarrow \infty$. Since $\left\|x_{n}-y_{n}\right\| \rightarrow 0$ and $\left\|y_{n}-t_{n}\right\| \rightarrow 0$ as $n \rightarrow \infty$, we also have $y_{n} \rightarrow p$ and $t_{n} \rightarrow p$ as $n \rightarrow \infty$. The proof is now complete.

\section{Applications}

In this section, we present some applications of our main result.

Using Theorem 2, we state a strong convergence theorem for the common fixed 
AN EXTRAGRADIENT ITERATIVE SCHEME FOR COMMON FIXED POINT PROBLEMS AND VARIATIONAL INEQUALITY PROBLEMS WITH

point of a sequence of asymptotically nonexpansive mappings and a strictly pseudocontractive mapping.

Theorem 3. Let $C$ be a nonempty closed convex subset of a real Hilbert space $H$. Let $T: C \rightarrow C$ be a $\lambda$-strictly pseudocontractive and $\mathcal{S}:=\left\{S_{n}\right\}$ a sequence of asymptotically nonexpansive mappings from $C$ into itself with sequence $\left\{k_{n}\right\}$ such that $F(\mathcal{S}) \cap F(T) \neq \emptyset$. Assume that $\mathcal{S}$ satisfies condition (D) and $f: C \rightarrow C$ is a $k$ - contraction. For arbitrary $x_{1} \in C$, consider the sequences $\left\{x_{n}\right\}$ and $\left\{y_{n}\right\}$ generated by the following iterative process:

$\left\{\begin{array}{l}x_{1} \in C, \\ y_{n}=\left(1-\lambda_{n}\right) x_{n}+\lambda_{n} T x_{n}, \\ x_{n+1}=\alpha_{n} f\left(x_{n}\right)+\left(1-\alpha_{n}\right) S_{n}\left(x_{n}-\lambda_{n}\left(y_{n}-T y_{n}\right)\right), \quad \text { for all } n \in \mathbb{N},\end{array}\right.$

where $\left\{\alpha_{n}\right\}$ and $\left\{\lambda_{n}\right\}$ are two sequences of positive numbers with $\left\{\alpha_{n}\right\} \subset(0, \bar{\alpha})$ and $\left\{\lambda_{n}\right\} \subset[a, b]$, with $0<a<b<\alpha(1-\delta)$ (for some $\bar{\alpha}, \delta \in(0,1)$ ) satisfying the conditions (i)- ( $v$ ) of Theorem 2. Then, the sequences $\left\{x_{n}\right\}$ and $\left\{y_{n}\right\}$ converge strongly to the same point $p$, such that $p$ is the unique solution in $F(\mathcal{S}) \cap F(T)$ of the following variational inequality:

$$
\langle f(p)-p, y-p\rangle \leq 0, \quad \text { for all } y \in F(\mathcal{S}) \cap F(T) .
$$

Proof. Put $A:=I-T$ in Theorem 2. Then $A$ is $\frac{1-\lambda}{2}$-inverse strongly monotone. We have that $F(T)=\Omega, P_{C}\left(x_{n}-\lambda_{n} A x_{n}\right)=x_{n}-\lambda_{n} A x_{n}=\left(1-\lambda_{n}\right) x_{n}+$ $\lambda_{n} T x_{n}$ and $P_{C}\left(x_{n}-\lambda_{n} A y_{n}\right)=x_{n}-\lambda_{n}\left(y_{n}-T y_{n}\right)$. So, by Theorem 2, we obtain the desired result.

Theorem 3 extends the results of Theorem 3.1 of Petruşel and Yao [10] and Theorem 4.1 of Chen, Zhang and Fan [2].

The following theorem extends the results of Theorem 3.2 of Petruşel and Yao [10] and Theorem 4.1 of Zeng and Yao [18].

Theorem 4. Let $H$ be a real Hilbert space, $A: H \rightarrow H$ be an $\alpha$-inverse strongly monotone mapping and $\mathcal{S}:=\left\{S_{n}\right\}$ a sequence of asymptotically nonexpansive mappings from $H$ into itself with sequence $\left\{k_{n}\right\}$ such that $F(\mathcal{S}) \cap$ $A^{-1}(0) \neq \emptyset$. Assume that $\mathcal{S}$ satisfies condition (D) and $f: C \rightarrow C$ is a $k$-contraction. For arbitrary $x_{1} \in C$, consider the sequences $\left\{x_{n}\right\}$ and $\left\{y_{n}\right\}$ generated by the following iterative process:

$\left\{\begin{array}{l}x_{1} \in C, \\ y_{n}=x_{n}-\lambda_{n} A x_{n}, \\ x_{n+1}=\alpha_{n} f\left(x_{n}\right)+\left(1-\alpha_{n}\right) S_{n}\left(x_{n}-\lambda_{n} A y_{n}\right), \quad \text { for all } n \in \mathbb{N},\end{array}\right.$ 
AN EXTRAGRADIENT ITERATIVE SCHEME FOR COMMON FIXED POINT PROBLEMS AND VARIATIONAL INEQUALITY PROBLEMS WITH

where $\left\{\alpha_{n}\right\}$ and $\left\{\lambda_{n}\right\}$ are two sequences of positive numbers with $\left\{\alpha_{n}\right\} \subset(0, \bar{\alpha})$ and $\left\{\lambda_{n}\right\} \subset[a, b]$, with $0<a<b<\alpha(1-\delta)$ (for some $\bar{\alpha}, \delta \in(0,1)$ ) satisfying the conditions (i)- (v) of Theorem 2. Then, the sequences $\left\{x_{n}\right\}$ and $\left\{y_{n}\right\}$ converge strongly to the same point $p$, such that $p$ is the unique solution in $F(\mathcal{S}) \cap A^{-1}(0)$ of the following variational inequality:

$$
\langle f(p)-p, y-p\rangle \leq 0, \quad \text { for all } y \in F(\mathcal{S}) \cap A^{-1}(0) .
$$

Proof. We have $A^{-1}(0)=\Omega$ and $P_{H}=I$. The conclusion follows from Theorem 2.

Theorem 5 extends Theorem 3.3 of Petruşel and Yao [10] and Theorem 4.2 of Zeng and Yao [18].

Theorem 5. Let $H$ be a real Hilbert space and $A: H \rightarrow H$ be an $\alpha$-inverse strongly monotone mapping. For each $n \in \mathbb{N}$, let $B_{n}$ be a maximal monotone operator from $H$ into $2^{H}$ with resolvent operator $J_{r}^{B_{n}}$ for some $r>0$ such that $F(\mathcal{J}) \cap A^{-1}(0) \neq \emptyset$. Assume that $\mathcal{J}=\left\{J_{r}^{B_{n}}\right\}$ satisfies condition (D) and $f: C \rightarrow C$ is a $k$-contraction. For arbitrary $x_{1} \in C$, consider the sequences $\left\{x_{n}\right\}$ and $\left\{y_{n}\right\}$ generated by the following iterative process:

$\left\{\begin{array}{l}x_{1} \in H, \\ y_{n}=x_{n}-\lambda_{n} A x_{n}, \\ x_{n+1}=\alpha_{n} f\left(x_{n}\right)+\left(1-\alpha_{n}\right) J_{r}^{B_{n}}\left(x_{n}-\lambda_{n} A y_{n}\right), \quad \text { for all } n \in \mathbb{N},\end{array}\right.$

where $\left\{\alpha_{n}\right\}$ and $\left\{\lambda_{n}\right\}$ are two sequences of positive numbers with $\left\{\alpha_{n}\right\} \subset(0, \bar{\alpha})$ and $\left\{\lambda_{n}\right\} \subset[a, b]$, with $0<a<b<\alpha(1-\delta)$ (for some $\bar{\alpha}, \delta \in(0,1)$ ) satisfying the conditions (i)- (iii) of Theorem 2 and (iv)':

(iv) $\lim _{n \rightarrow \infty} \frac{\left\|J_{r}^{B_{n}} t_{n}-J_{r}^{B_{n+1}} t_{n}\right\|}{\alpha_{n+1}}=0$.

Then, the sequences $\left\{x_{n}\right\}$ and $\left\{y_{n}\right\}$ converge strongly to the same point $p \in$ $F(\mathcal{J}) \cap A^{-1}(0)$, where $p$ is the unique solution in $F(\mathcal{J}) \cap A^{-1}(0)$ of the following variational inequality:

$$
\langle f(p)-p, y-p\rangle \leq 0, \quad \text { for all } y \in F(\mathcal{J}) \cap A^{-1}(0) .
$$

Proof. We have $A^{-1}(0)=\Omega$. The conclusion follows from Theorem 2, by putting $P_{H}=I$ and $J_{r}^{B_{n}}=S_{n}$.

We now impose some condition on $\mathcal{S}$ to fulfill condition $(\mathcal{D})$ in Theorem 2. 
AN EXTRAGRADIENT ITERATIVE SCHEME FOR COMMON FIXED POINT PROBLEMS AND VARIATIONAL INEQUALITY PROBLEMS WITH

Theorem 6. Let $C$ be a nonempty, closed and convex subset of a real Hilbert space $H$. Let $A: C \rightarrow H$ be an $\alpha$-inverse strongly monotone mapping and $S$ an asymptotically nonexpansive mapping from $C$ into itself with sequence $\left\{k_{n}\right\}$ such that $F(S) \cap \Omega \neq \emptyset$ and $f: C \rightarrow C$ a $k$-contraction. For arbitrary $x_{1} \in C$, consider the sequences $\left\{x_{n}\right\}$ and $\left\{y_{n}\right\}$ generated by the following iterative process:

$$
\left\{\begin{array}{l}
x_{1} \in C, \\
y_{n}=P_{C}\left(x_{n}-\lambda_{n} A x_{n}\right), \\
x_{n+1}=\alpha_{n} f\left(x_{n}\right)+\left(1-\alpha_{n}\right) S^{n} P_{C}\left(x_{n}-\lambda_{n} A y_{n}\right), \quad \text { for all } n \in \mathbb{N},
\end{array}\right.
$$

where $\left\{\alpha_{n}\right\}$ and $\left\{\lambda_{n}\right\}$ are two sequences of positive numbers with $\left\{\alpha_{n}\right\} \subset(0, \bar{\alpha})$ and $\left\{\lambda_{n}\right\} \subset[a, b]$, with $0<a<b<\alpha(1-\delta$ ) (for some $\bar{\alpha}, \delta \in(0,1)$ ) satisfying the conditions (i)- (v) of Theorem 2 with $S_{n}=S^{n}$. Then, the sequences $\left\{x_{n}\right\}$ and $\left\{y_{n}\right\}$ converge strongly to the same point $p$, such that $p$ is the unique solution in $F(S) \cap \Omega$ of the following variational inequality:

$$
\langle f(p)-p, y-p\rangle \leq 0, \quad \text { for all } y \in F(S) \cap \Omega .
$$

Proof. It is sufficient to show that $\left\{S^{n}: n \in \mathbb{N}\right\}$ holds condition $(\mathcal{D})$. Observe that

$$
\begin{aligned}
\left\|x_{n}-S x_{n}\right\| \leq & \left\|x_{n}-x_{n+1}\right\|+\left\|x_{n+1}-S^{n} t_{n}\right\| \\
& +\left\|S^{n} t_{n}-S^{n+1} t_{n}\right\|+\left\|S^{n+1} t_{n}-S x_{n}\right\| \\
\leq & \left\|x_{n}-x_{n+1}\right\|+\left\|x_{n+1}-S^{n} t_{n}\right\|+\left\|S^{n} t_{n}-S^{n+1} t_{n}\right\| \\
& +k_{1}\left(\left\|S^{n} t_{n}-x_{n+1}\right\|+\left\|x_{n+1}-x_{n}\right\|\right) \rightarrow 0 \text { as } n \rightarrow \infty .
\end{aligned}
$$

One can see by Lemma 4, that condition $(\mathcal{D})$ holds.

We now derive the main result of Petruşel and Yao ([10], Theorem 2.2) as Corollary.

Corollary 1. Let $C$ be a nonempty, closed and convex subset of a real Hilbert space $H$. Let $A: C \rightarrow H$ be an $\alpha$-inverse strongly monotone mapping and $S: C \rightarrow C$ a nonexpansive mapping such that $F(S) \cap \Omega \neq \emptyset$. Let $\left\{\alpha_{n}\right\}$ and $\left\{\lambda_{n}\right\}$ be two sequences of positive numbers with $\left\{\alpha_{n}\right\} \subset(0,1)$ and $\left\{\lambda_{n}\right\} \subset[a, b]$, with $0<a<b<\alpha(1-\delta)$ (for some $\delta \in(0,1)$ ) satisfying the conditions:

(i) $\lim _{n \rightarrow \infty} \alpha_{n}=0, \sum_{n=1}^{\infty} \alpha_{n}=\infty$;

(ii) $\sum_{n=1}^{\infty}\left|\alpha_{n+1}-\alpha_{n}\right|<\infty$;

(iii) $\sum_{n=1}^{\infty}\left|\lambda_{n+1}-\lambda_{n}\right|<\infty$; 
AN EXTRAGRADIENT ITERATIVE SCHEME FOR COMMON FIXED POINT PROBLEMS AND VARIATIONAL INEQUALITY PROBLEMS WITH

For arbitrary $x_{1} \in C$, consider the sequences $\left\{x_{n}\right\}$ and $\left\{y_{n}\right\}$ generated by the following iterative process:

$$
\left\{\begin{array}{l}
x_{1} \in C, \\
y_{n}=P_{C}\left(x_{n}-\lambda_{n} A x_{n}\right), \\
x_{n+1}=\alpha_{n} f\left(x_{n}\right)+\left(1-\alpha_{n}\right) S P_{C}\left(x_{n}-\lambda_{n} A y_{n}\right), \quad \text { for all } n \in \mathbb{N},
\end{array}\right.
$$

where $f: C \rightarrow C$ is a k-contraction.

Then, the sequences $\left\{x_{n}\right\}$ and $\left\{y_{n}\right\}$ converge strongly to the same point $p$, such that $p$ is the unique solution in $F(S) \cap \Omega$ of the following variational inequality:

$$
\langle f(p)-p, y-p\rangle \leq 0, \quad \text { for all } y \in F(S) \cap \Omega .
$$

Example. Let $H=C=\mathbb{R}$. Let $A, f: C \rightarrow C$ be mappings defined by $A(x)=\frac{x}{2}$ and $f(x)=\frac{3 x}{16}, \forall x \in C$. Then $A$ is 2-inverse strongly monotone mapping and $f$ is a contraction mapping. Let $\mathcal{S}:=\left\{S_{n}\right\}$ be a sequence of asymptotically nonexpansive mappings from $C$ into $C$ defined by $S_{n}(x)=$ $\left(1+\frac{1}{n}\right) x, \forall x \in C$ and $n \in \mathbb{N}$. Clearly, $F(\mathcal{S})=\{0\}$ and $F(\mathcal{S}) \cap \Omega=\{0\}$. Let $\left(\alpha_{n}\right)_{n \in \mathbb{N}}$ and $\left(\lambda_{n}\right)_{n \in \mathbb{N}}$ be two sequences of positive numbers defined by $\alpha_{n}=\frac{1}{n+1}$ and $\lambda_{n}=\frac{1}{2}$.

Then, the sequence $\left\{x_{n}\right\}$ generated by

$$
\left\{\begin{array}{l}
x_{1} \in C, \\
y_{n}=x_{n}-\lambda_{n} A x_{n}, \\
x_{n+1}=\alpha_{n} f\left(x_{n}\right)+\left(1-\alpha_{n}\right) S_{n}\left(x_{n}-\lambda_{n} A y_{n}\right), \quad \text { for all } n \in \mathbb{N},
\end{array}\right.
$$

satisfying the inequality:

$$
x_{n+1}=\left[\frac{3}{n+1}+13\right] \frac{x_{n}}{16} \leq \frac{29}{32} x_{n} .
$$

One can see easily that $\left\{x_{n}\right\}$ converges to $0 \in F(\mathcal{S}) \cap \Omega$.

Remark 1. For the numerical simulation and the graphic representation of the above sequences, see Figure 1 and Figure 2 below. 
AN EXTRAGRADIENT ITERATIVE SCHEME FOR COMMON FIXED POINT PROBLEMS AND VARIATIONAL INEQUALITY PROBLEMS WITH

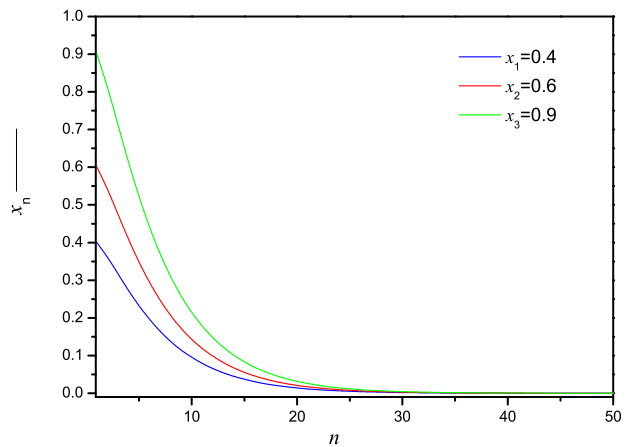

Figure 1: Convergence of the sequence $\left\{x_{n}\right\}$ for $n$ iterations.

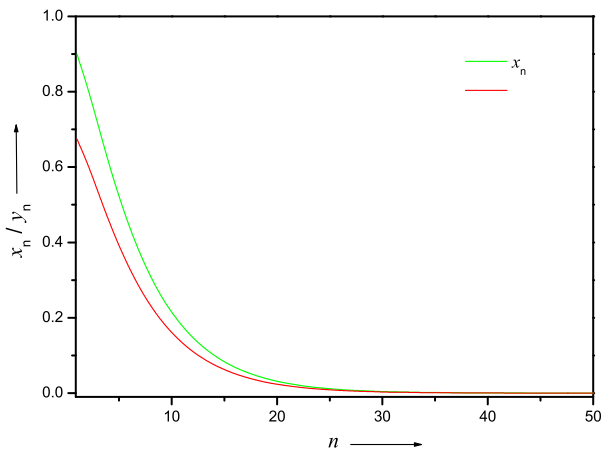

Figure 2: Convergence of the sequences $\left\{x_{n}\right\}$ and $\left\{y_{n}\right\}$ for $n$ iterations. 
AN EXTRAGRADIENT ITERATIVE SCHEME FOR COMMON FIXED POINT PROBLEMS AND VARIATIONAL INEQUALITY PROBLEMS WITH

\section{References}

[1] R.P. Agarwal, Donal O'Regan and D.R. Sahu, Fixed Point Theory for Lipschitzian-type Mappings with Applications, Series: Topological Fixed Point Theory and Its Applications Springer, New York, 2009.

[2] J. Chen, L. Zhang and T. Fan, Viscosity approximation methods for nonexpansive mappings and monotone mappings, J. Math. Anal. Appl. 334 (2007), 1450-1461.

[3] Y.J. Cho, H. Zhou and G. Guo, Weak and strong convergence theorems for three-step iterations with errors for asymptotically nonexpansive mappings, Comput. Math. Appl., 47 (2004), 707-717.

[4] Q.-L. Dong and S. He, A viscosity projection method for class $\mathcal{T}$ mappings, Analele Ştiinţifice ale Univ. Ovidius Constanţa 21 (2013), 95-109.

[5] K. Goebel and W.A. Kirk, Topics on Metric Fixed Point Theory, Cambridge University Press, Cambridge, 1990.

[6] G.M. Korpelevich, An extragradient method for finding saddle points and for other problems, Ekonomika i Matematicheskie Metody 12 (1976), 747756.

[7] N. Nadezhkina and W. Takahashi, Weak convergence theorem by an extragradient method for nonexpansive mappings and monotone mappings, J. Optim. Theory Appl., 128 (2006), 191-201.

[8] M.A. Noor, Modified projection method for pseudomonotone variational inequalities, Appl. Math. Lett. 15 (2002), 315-320.

[9] Z. Opial, Weak convergence of the sequence of successive approximations for nonexpansive mappings, Bull. Amer. Math. Soc. 73 (1967), 591-597.

[10] A. Petruşel and J.-C. Yao, An extragradient iterative scheme by viscosity approximation methods for fixed point problems and variational inequality problems, Cent. Eur. J. Math. 7 (2009), 335-347.

[11] R.T. Rockafellar, On the maximality of sums of nonlinear monotone operators, Trans. Amer. Math. Soc. 149 (1970), 75-88.

[12] D.R. Sahu, Zequing Liu, and Shin Min Kang, Iterative approaches to common fixed points of asymptotically nonexpansive mappings, Rocky Mountain J. Math. 39 (2009), 281-304. 
AN EXTRAGRADIENT ITERATIVE SCHEME FOR COMMON FIXED POINT PROBLEMS AND VARIATIONAL INEQUALITY PROBLEMS WITH

[13] J. Schu, Weak and strong convergence to fixed points of asymptotically nonexpansive mappings, Bull. Austral. Math. Soc. 43 (1991), 153-159.

[14] G. Stampacchia, Formes bilineaires coercivities sur les ensembles convexes, C. R. Acad. Sci. Paris 258 (1964), 4413-4416.

[15] W. Takahashi, Nonlinear Functional Analysis, Yokohama Publishers, Yokohama, Japan, 2000.

[16] W. Takahashi and M. Toyoda, Weak convergence theorems for nonexpansive mappings and monotone mappings, J. Optim. Theory Appl. 118 (2003), 417-428.

[17] H.K. Xu and T.H. Kim, Convergence of hybrid sleepest-descend methods for variational inequalities, J. Optim. Theory Appl. 119 (2003), 185-201.

[18] L.C. Zeng and J.-C. Yao, Strong convergence theorem by an extragradient method for fixed point problems and variational inequality problems, Taiwan. J. Math., 10 (2006), 1293-1303.

Adrian PETRUSEL,

Department of Mathematics,

Babeş-Bolyai University Cluj-Napoca,

Kogălniceanu Street, No. 1, 400084, Cluj-Napoca, Romania.

Email: petrusel@math.ubbcluj.ro

D.R. SAHU,

Department of Mathematics,

Banaras Hindu University,

Varanasi-221005, India.

Email: drsahudr@gmail.com

Vidya SAGAR,

Department of Mathematics,

Banaras Hindu University,

Varanasi-221005, India.

Email: luckvidya@yahoo.com 